\title{
LOCAL CLASSIFICATION AND EXAMPLES OF AN IMPORTANT CLASS OF PARACONTACT METRIC MANIFOLDS
}

\author{
VERÓNICA MARTÍN-MOLINA
}

\begin{abstract}
We study paracontact metric $(\kappa, \mu)$-spaces with $\kappa=-1$, equivalent to $h^{2}=0$ but not $h=0$. In particular, we will give an alternative proof of Theorem 3.2 of [11] and present examples of paracontact metric $(-1,2)$-spaces and $(-1,0)$-spaces of arbitrary dimension with tensor $h$ of every possible constant rank. We will also show explicit examples of paracontact metric $(-1, \mu)$ spaces with tensor $h$ of non-constant rank, which were not known to exist until now.
\end{abstract}

\section{INTRODUCTION}

Paracontact metric manifolds, the odd-dimensional analogue of paraHermitian manifolds, were first introduced in [10] and they have been the object of intense study recently, particularly since the publication of [14]. An important class among paracontact metric manifolds is that of the $(\kappa, \mu)$-spaces, which satisfy the nullity condition $[5$

$$
R(X, Y) \xi=\kappa(\eta(Y) X-\eta(X) Y)+\mu(\eta(Y) h X-\eta(X) h Y),
$$

for all $X, Y$ vector fields on $M$, where $\kappa$ and $\mu$ are constants and $h=\frac{1}{2} L_{\xi} \varphi$.

This class includes the paraSasakian manifolds 10, 14, the paracontact metric manifolds satisfying $R(X, Y) \xi=0$ for all $X, Y$ [15], certain $g$-natural paracontact metric structures constructed on unit tangent sphere bundles [7, etc.

The definition of a paracontact metric $(\kappa, \mu)$-space was motivated by the relationship between contact metric and paracontact geometry. More precisely, it was proved in [4 that any non-Sasakian contact metric $(\kappa, \mu)$-space accepts two paracontact metric $(\widetilde{\kappa}, \widetilde{\mu})$-structures with the same contact form. On the other hand, under certain natural conditions, every non-paraSasakian paracontact $(\widetilde{\kappa}, \widetilde{\mu})$-space admits a contact metric $(\kappa, \mu)$-structure compatible with the same contact form ([5]).

Paracontact metric $(\kappa, \mu)$-spaces satisfy that $h^{2}=(\kappa+1) \phi^{2}$ but this condition does not give any type of restriction over the value of $\kappa$, unlike in contact metric geometry, because the metric of a paracontact metric manifold is not positive definite. However, it is useful to distinguish the cases $\kappa>-1, \kappa<-1$ and $\kappa=-1$. In the first two, equation (1) determines the curvature completely and either the tensor $h$ or $\varphi h$ are diagonalisable [5. The case $\kappa=-1$ is equivalent to $h^{2}=0$ but not to $h=0$. Indeed, there are examples of paracontact metric $(\kappa, \mu)$-spaces with $h^{2}=0$ but $h \neq 0$, as was first shown in [2, 5, 8, 12].

However, only some particular examples were given of this type of space and no effort had been made to understand the general behaviour of the tensor $h$ of a paracontact metric $(-1, \mu)$-space until the author published [11, where a local classification depending on the rank of $h$ was given in Theorem 3.2. The author also provided explicit examples of all the possible constant values of the rank of $h$ when $\mu=2$. She explained why the values $\mu=0$ and $\mu=2$ are special and studying them is enough. Finally, she showed some paracontact metric $(-1,0)$-spaces of any dimension with

2010 Mathematics Subject Classification. Primary 53C15, 53B30; Secondary 53C25, 53C50.

Key words and phrases. paracontact metric manifold; $(\kappa, \mu)$-spaces; paraSasakian; contact metric; nullity distribution.

The author is is partially supported by the PAI group FQM-327 (Junta de Andalucía, Spain), the group Geometría E15 (Gobierno de Aragón, Spain), the MINECO grant MTM2011-22621 and the "Centro Universitario de la Defensa de Zaragoza" grant ID2013-15. 
$\operatorname{rank}(h)=1$ and of paracontact metric $(-1,0)$-spaces of dimension 5 and 7 for any possible constant rank of $h$. These were the first examples of this type with $\mu \neq 2$ and dimension greater than 3 .

In the present paper, after the preliminaries section, we will give an alternative proof of Theorem 3.2 of [11] that does not use [13] and we will complete the examples of all the possible cases of constant rank of $h$ by presenting $(2 n+1)$-dimensional paracontact metric $(-1,0)$-spaces with $\operatorname{rank}(h)=2, \ldots, n$. Lastly, we will also show the first explicit examples ever known of paracontact metric $(-1,2)$-spaces and $(-1,0)$-spaces with $h$ of non-constant rank.

\section{Preliminaries}

An almost paracontact structure on a $(2 n+1)$-dimensional smooth manifold $M$ is given by a $(1,1)$-tensor field $\varphi$, a vector field $\xi$ and a 1-form $\eta$ satisfying the following conditions [10]:

(i) $\eta(\xi)=1, \varphi^{2}=I-\eta \otimes \xi$,

(ii) the eigendistributions $\mathcal{D}^{+}$and $\mathcal{D}^{-}$of $\varphi$ corresponding to the eigenvalues 1 and -1 , respectively, have equal dimension $n$.

It follows that $\varphi \xi=0, \eta \circ \varphi=0$ and $\operatorname{rank}(\varphi)=2 n$. If an almost paracontact manifold admits a semi-Riemannian metric $g$ such that

$$
g(\varphi X, \varphi Y)=-g(X, Y)+\eta(X) \eta(Y),
$$

for all $X, Y$ on $M$, then $(M, \varphi, \xi, \eta, g)$ is called an almost paracontact metric manifold. Then $g$ is necessarily of signature $(n+1, n)$ and satisfies $\eta=g(\cdot, \xi)$ and $g(\cdot, \varphi \cdot)=-g(\varphi \cdot, \cdot)$.

We can now define the fundamental 2 -form of the almost paracontact metric manifold by $\Phi(X, Y)=$ $g(X, \varphi Y)$. If $d \eta=\Phi$, then $\eta$ becomes a contact form (i.e. $\left.\eta \wedge(d \eta)^{n} \neq 0\right)$ and $(M, \varphi, \xi, \eta, g)$ is said to be a paracontact metric manifold.

We can also define on a paracontact metric manifold the tensor field $h:=\frac{1}{2} L_{\xi} \varphi$, which is symmetric with respect to $g$ (i.e. $g(h X, Y)=g(X, h Y)$, for all $X, Y)$, anti-commutes with $\varphi$ and satisfies $h \xi=\operatorname{tr} h=0$ and the identity $\nabla \xi=-\varphi+\varphi h(14)$. Moreover, it vanishes identically if and only if $\xi$ is a Killing vector field, in which case $(M, \varphi, \xi, \eta, g)$ is called a $K$-paracontact manifold.

An almost paracontact structure is said to be normal if and only if the tensor $[\varphi, \varphi]-2 d \eta \otimes \xi=0$, where $[\varphi, \varphi]$ is the Nijenhuis tensor of $\varphi[14$ :

$$
[\varphi, \varphi](X, Y)=\varphi^{2}[X, Y]+[\varphi X, \varphi Y]-\varphi[\varphi X, Y]-\varphi[X, \varphi Y] .
$$

A normal paracontact metric manifold is said to be a paraSasakian manifold and is in particular $K$-paracontact. The converse holds in dimension 3 ([6] $)$ but not in general in higher dimensions. However, it was proved in Theorem 3.1 of [11] that it also holds for $(-1, \mu)$-spaces. Every paraSasakian manifold satisfies

$$
R(X, Y) \xi=-(\eta(Y) X-\eta(X) Y),
$$

for every $X, Y$ on $M$. The converse is not true, since Examples 3.8-3.11 of [11] and Examples 4.1 and 4.5 of the present one show that there are examples of paracontact metric manifolds satisfying equation (2) but with $h \neq 0$ (and therefore not K-paracontact or paraSasakian). Moreover, it is also clear in Example 4.5 that the rank of $h$ does not need to be constant either, since $h$ can be zero at some points and non-zero in others.

The main result of [11] is the following local classification of paracontact metric $(-1, \mu)$-spaces:

Theorem 2.1 ([1]). Let $M$ be a $(2 n+1)$-dimensional paracontact metric $(-1, \mu)$-space. Then we have one of the following possibilities:

(1) either $h=0$ and $M$ is paraSasakian,

(2) or $h \neq 0$ and $\operatorname{rank}\left(h_{p}\right) \in\{1, \ldots, n\}$ at every $p \in M$ where $h_{p} \neq 0$. Moreover, there exists a basis $\left\{\xi_{p}, X_{1}, Y_{1}, \ldots, X_{n}, Y_{n}\right\}$ of $T_{p}(M)$ such that the only non-vanishing components of $g$ are

$$
g_{p}\left(\xi_{p}, \xi_{p}\right)=1, \quad g_{p}\left(X_{i}, Y_{i}\right)= \pm 1
$$


and

$$
h_{p \mid\left\langle X_{i}, Y_{i}\right\rangle}=\left(\begin{array}{ll}
0 & 0 \\
1 & 0
\end{array}\right) \quad \text { or } \quad h_{p \mid\left\langle X_{i}, Y_{i}\right\rangle}=\left(\begin{array}{ll}
0 & 0 \\
0 & 0
\end{array}\right),
$$

where obviously there are exactly rank $\left(h_{p}\right)$ submatrices of the first type.

If $n=1$, such a basis $\left\{\xi_{p}, X_{1}, Y_{1}\right\}$ also satisfies that

$$
\varphi_{p} X_{1}= \pm X_{1}, \quad \varphi_{p} Y_{1}=\mp Y_{1},
$$

and the tensor $h$ can be written as

$$
h_{p \mid\left\langle\xi_{p}, X_{1}, Y_{1}\right\rangle}=\left(\begin{array}{ccc}
0 & 0 & 0 \\
0 & 0 & 0 \\
0 & 1 & 0
\end{array}\right) .
$$

Many examples of paraSasakian manifolds are known. For instance, hyperboloids $\mathbb{H}_{n+1}^{2 n+1}(1)$ and the hyperbolic Heisenberg group $\mathcal{H}^{2 n+1}=\mathbb{R}^{2 n} \times \mathbb{R}, 9$. We can also obtain $(\eta$-Einstein) paraSasakian manifolds from contact $(\kappa, \mu)$-spaces with $\left|1-\frac{\mu}{2}\right|<\sqrt{1-\kappa}$. In particular, the tangent sphere bundle $T_{1} N$ of any space form $N(c)$ with $c<0$ admits a canonical $\eta$-Einstein paraSasakian structure, 3 . Finally, we can see how to construct explicitly a paraSasakian structure on a Lie group (see Example 3.4 of [1] ) or on the unit tangent sphere bundle, [7].

On the other hand, until [1] only some types of non-paraSasakian paracontact metric $(-1, \mu)-$ spaces were known:

- $(2 n+1)$-dimensional paracontact metric $(-1,2)$-space with $\operatorname{rank}(h)=n,[5]$.

- 3-dimensional paracontact metric $(-1,2)$-space with $\operatorname{rank}(h)=n=1,[12$.

- 3-dimensional paracontact metric $(-1,0)$-space with $\operatorname{rank}(h)=n=1$. This example is not paraSasakian but it satisfies (2), 8].

The answer to why there seems to be only examples of paracontact metric $(-1, \mu)$-spaces with $\mu=2$ or $\mu=0$ is a $\mathcal{D}_{c}$-homothetic deformation, i.e. the following change of a paracontact metric structure $(M, \varphi, \xi, \eta, g)[14$ :

$$
\varphi^{\prime}:=\varphi, \quad \xi^{\prime}:=\frac{1}{c} \xi, \quad \eta^{\prime}:=c \eta, \quad g^{\prime}:=c g+c(c-1) \eta \otimes \eta,
$$

for some $c \neq 0$.

It is known that $\left(\varphi^{\prime}, \xi^{\prime}, \eta^{\prime}, g^{\prime}\right)$ is again a paracontact metric structure on $M$ and that $K$-paracontact and paraSasakian structures are also preserved. However, curvature conditions like $R(X, Y) \xi=0$ are destroyed, since paracontact metric $(\kappa, \mu)$-spaces become other paracontact metric $\left(\kappa^{\prime}, \mu^{\prime}\right)$-spaces with

$$
\kappa^{\prime}=\frac{\kappa+1-c^{2}}{c^{2}}, \quad \mu^{\prime}=\frac{\mu-2+2 c}{c} .
$$

In particular, if $(M, \varphi, \xi, \eta, g)$ is a paracontact metric $(-1, \mu)$-space, then the deformed manifold is another paracontact metric $\left(-1, \mu^{\prime}\right)$-space with $\mu^{\prime}=\frac{\mu-2+2 c}{c}$.

Therefore, given a $(-1,2)$-space, a $\mathcal{D}_{c}$-homothetic deformation with arbitrary $c \neq 0$ will give us another paracontact metric $(-1,2)$-space. Given a paracontact metric $(-1,0)$-space, if we $\mathcal{D}_{c^{-}}$ homothetically deform it with $c=\frac{2}{2-\mu} \neq 0$ for some $\mu \neq 2$, we will obtain a paracontact metric $(-1, \mu)$-space with $\mu \neq 2$. A sort of converse is also possible: given a $(-1, \mu)$-space with $\mu \neq 2$, a $\mathcal{D}_{c}$-homothetic deformation with $c=1-\frac{\mu}{2} \neq 0$ will give us a paracontact metric $(-1,0)$-space. The case $\mu=0, h \neq 0$ is also special because the manifold satisfies (2) but it is not paraSasakian.

Examples of non-paraSasakian paracontact metric $(-1,2)$-spaces of any possible dimension and constant rank of $h$ were presented in [11]:

Example $2.2((2 n+1)$-dimensional paracontact metric $(-1,2)$-space with $\operatorname{rank}(h)=m \in\{1, \ldots, n\})$. Let $\mathfrak{g}$ be the $(2 n+1)$-dimensional Lie algebra with basis $\left\{\xi, X_{1}, Y_{1}, \ldots, X_{n}, Y_{n}\right\}$ such that the only 
non-zero Lie brackets are:

$$
\begin{aligned}
{\left[\xi, X_{i}\right] } & =Y_{i}, \quad i=1, \ldots, m, \\
{\left[X_{i}, Y_{j}\right] } & = \begin{cases}\delta_{i j}\left(2 \xi+\sqrt{2}\left(1+\delta_{i m}\right) Y_{m}\right) \\
\quad+\left(1-\delta_{i j}\right) \sqrt{2}\left(\delta_{i m} Y_{j}+\delta_{j m} Y_{i}\right), & i, j=1, \ldots, m, \\
\delta_{i j}\left(2 \xi+\sqrt{2} Y_{i}\right), & i, j=m+1, \ldots, n, \\
\sqrt{2} Y_{i}, & i=1, \ldots, m, j=m+1, \ldots, n .\end{cases}
\end{aligned}
$$

If we denote by $G$ the Lie group whose Lie algebra is $\mathfrak{g}$, we can define a left-invariant paracontact metric structure on $G$ the following way:

$$
\begin{gathered}
\varphi \xi=0, \quad \varphi X_{i}=X_{i}, \quad \varphi Y_{i}=-Y_{i}, \quad i=1, \ldots, n, \\
\eta(\xi)=1, \quad \eta\left(X_{i}\right)=\eta\left(Y_{i}\right)=0, \quad i=1, \ldots, n .
\end{gathered}
$$

The only non-vanishing components of the metric are

$$
g(\xi, \xi)=g\left(X_{i}, Y_{i}\right)=1, \quad i=1, \ldots, n .
$$

A straightforward computation gives that $h X_{i}=Y_{i}$ if $i=1, \ldots, m, h X_{i}=0$ if $i=m+1, \ldots, n$ and $h Y_{j}=0$ if $j=1, \ldots, n$, so $h^{2}=0$ and rank $(h)=m$. Furthermore, the manifold is a $(-1,2)$-space.

Examples of non-paraSasakian paracontact metric $(-1,0)$-spaces of any possible dimension and $\operatorname{rank}(h)=1$ were also given in [1]:

Example $2.3((2 n+1)$-dimensional paracontact metric $(-1,0)$-space with $\operatorname{rank}(h)=1)$. Let $\mathfrak{g}$ be the $(2 n+1)$-dimensional Lie algebra with basis $\left\{\xi, X_{1}, Y_{1}, \ldots, X_{n}, Y_{n}\right\}$ such that the only non-zero Lie brackets are:

$$
\begin{aligned}
{\left[\xi, X_{1}\right] } & =X_{1}+Y_{1}, & {\left[\xi, Y_{1}\right] } & =-Y_{1}, & {\left[X_{1}, Y_{1}\right] } & =2 \xi \\
{\left[X_{i}, Y_{i}\right] } & =2\left(\xi+Y_{i}\right), & {\left[X_{1}, Y_{i}\right] } & =X_{1}+Y_{1}, & {\left[Y_{1}, Y_{i}\right] } & =-Y_{1}, \quad i=2, \ldots, n .
\end{aligned}
$$

If we denote by $G$ the Lie group whose Lie algebra is $\mathfrak{g}$, we can define a left-invariant paracontact metric structure on $G$ the following way:

$$
\begin{gathered}
\varphi \xi=0, \quad \varphi X_{1}=X_{1}, \quad \varphi Y_{1}=-Y_{1}, \quad \varphi X_{i}=-X_{i}, \quad \varphi Y_{i}=Y_{i}, \quad i=2, \ldots, n, \\
\eta(\xi)=1, \quad \eta\left(X_{i}\right)=\eta\left(Y_{i}\right)=0, \quad i=1, \ldots, n .
\end{gathered}
$$

The only non-vanishing components of the metric are

$$
g(\xi, \xi)=g\left(X_{1}, Y_{1}\right)=1, \quad g\left(X_{i}, Y_{i}\right)=-1, \quad i=2, \ldots, n .
$$

$A$ straightforward computation gives that $h X_{1}=Y_{1}, h Y_{1}=0$ and $h X_{i}=h Y_{i}=0, i=2, \ldots, n$, so $h^{2}=0$ and $\operatorname{rank}(h)=1$.

Moreover, by basic paracontact metric properties and Koszul's formula we obtain that

$$
\begin{gathered}
\nabla_{\xi} X_{1}=0, \quad \nabla_{\xi} Y_{1}=0, \quad \nabla_{\xi} X_{i}=X_{i}, \quad \nabla_{\xi} Y_{i}=-Y_{i}, \quad i=2, \ldots, n, \\
\nabla_{X_{i}} Y_{1}=\delta_{i 1} \xi, \quad \nabla_{X_{i}} Y_{j}=\delta_{i j}\left(\xi+2 Y_{i}\right), \quad \nabla_{Y_{1}} X_{1}=-\xi, \quad \nabla_{Y_{i}} X_{j}=-\delta_{i j} \xi, \quad i, j=2, \ldots, n, \\
\nabla_{X_{1}} X_{j}=0, \quad \nabla_{Y_{1}} Y_{1}=\nabla_{Y_{1}} Y_{j}=0, \quad \nabla_{Y_{j}} Y_{1}=Y_{1}, \quad i=2, \ldots, n,
\end{gathered}
$$

and thus

$$
\begin{aligned}
R\left(X_{i}, \xi\right) \xi & =-X_{i}, \quad i=1, \ldots, n, \\
R\left(Y_{i}, \xi\right) \xi & =-Y_{i}, \quad i=1, \ldots, n, \\
R\left(X_{i}, X_{j}\right) \xi & =R\left(X_{i}, Y_{j}\right) \xi=R\left(Y_{i}, Y_{j}\right) \xi=0, \quad i, j=1, \ldots, n .
\end{aligned}
$$

Therefore, the manifold is also a $(-1,0)$-space. 
To our knowledge, the previous example is the first paracontact metric $(-1, \mu)$-space with $h^{2}=0$, $h \neq 0$ and $\mu \neq 2$ that was constructed in dimensions greater than 3. For dimension 3, Example 4.6 of 8 . was already known.

In dimension 5 , there also exist examples of paracontact metric $(-1,0)$-space with $\operatorname{rank}(h)=2$ and in dimension 7 of $\operatorname{rank}(h)=2,3$, as shown in 11. Higher-dimensional examples of paracontact metric $(-1,0)$-spaces with $\operatorname{rank}(h) \geq 2$ were not included, which will be remedied in Example 4.1 We will also see how to construct a 3 -dimensional paracontact metric $(-1,0)$-space and $(-1,2)$-space where the rank of $h$ is not constant.

\section{NeW Proof of Theorem 2.1}

We will now present a revised proof of Theorem 2.1 that does not use [13 when $h \neq 0$ but constructs the basis explicitly.

Proof. Since $\kappa=-1$, we know from [5] that $h^{2}=0$. If $h=0$, then $R(X, Y) \xi=-(\eta(Y) X-\eta(X) Y)$, for all $X, Y$ on $M$ and $\xi$ is a Killing vector field, so Theorem 3.1 of [11] gives us that the manifold is paraSasakian.

If $h \neq 0$, then let us take a point $p \in M$ such that $h_{p} \neq 0$. We know that $\xi$ is a global vector field such that $g(\xi, \xi)=1$, that $h \xi=0$ and that $h$ is self-adjoint, so $K e r \eta_{p}$ is $h$-invariant and $h_{p}: K e r \eta_{p} \mapsto K e r \eta_{p}$ is a non-zero linear map such that $h_{p}^{2}=0$. We will now construct a basis $\left\{X_{1}, Y_{1}, \ldots, X_{n}, Y_{n}\right\}$ of $\mathrm{Ker}_{p}$ that satisfies all of our requirements.

Take a non-zero vector $v \in K e r \eta_{p}$ such that $h_{p} v \neq 0$, which we know exists because $h_{p} \neq 0$. Then we write $K e r \eta_{p}=L_{1} \oplus L_{1}^{\perp}$, where $L_{1}=\left\langle v, h_{p} v\right\rangle$. Both $L_{1}$ and $L_{1}^{\perp}$ are $h_{p}$-invariant because $h_{p}$ is self-adjoint. Moreover, $g_{p}\left(v, h_{p} v\right) \neq 0$ because $g_{p}\left(h_{p} v, h_{p} v\right)=0=g_{p}\left(h_{p} v, w\right)$ for all $w \in L_{1}^{\perp}$, $h_{p} v \neq 0$ and $g$ is a non-degenerate metric. We now distinguish two cases:

(1) If $g_{p}(v, v)=0$, then we can take $X_{i}=\frac{1}{\sqrt{\left|g_{p}\left(v, h_{p} v\right)\right|}} v$ and $Y_{i}=\frac{1}{\sqrt{\left|g_{p}\left(v, h_{p} v\right)\right|}} h_{p} v$, which satisfy that $g_{p}\left(X_{i}, X_{i}\right)=0=g_{p}\left(Y_{i}, Y_{i}\right), g_{p}\left(X_{i}, Y_{i}\right)= \pm 1$ and $h_{p} X_{i}=Y_{i}$.

(2) If $g_{p}(v, v) \neq 0$, then $v^{\prime}=v-\frac{g_{p}(v, v)}{g_{p}\left(v, h_{p} v\right)} h_{p} v$ satisfies that $g_{p}\left(v^{\prime}, v^{\prime}\right)=0$, so we can take $X_{i}=\frac{1}{\sqrt{\left|g_{p}\left(v^{\prime}, h_{p} v\right)^{\prime}\right|}} v^{\prime}, Y_{i}=\frac{1}{\sqrt{\left|g_{p}\left(v^{\prime}, h_{p} v^{\prime}\right)\right|}} h v^{\prime}$. We have again that $g_{p}\left(X_{i}, X_{i}\right)=0=g_{p}\left(Y_{i}, Y_{i}\right)$, $g_{p}\left(X_{i}, Y_{i}\right)= \pm 1$ and $h_{p} X_{i}=Y_{i}$.

In both cases, $L_{1}=\left\langle X_{i}, Y_{i}\right\rangle$, so we now take a non-zero vector $v \in L_{1}^{\perp}$ and check if $h_{p} v \neq 0$. We know that we can take $v$ such that $h_{p} v \neq 0$ in this step as many times as the rank of $h_{p}$, which is at minimum 1 (since $h_{p} \neq 0$ ) and at most $n$ because $\operatorname{dim} K e r \eta_{p}=2 n$ and the spaces $L_{1}$ have dimension 2 .

If we denote by $m$ the rank of $h_{p}$, then we can write $K e r \eta_{p}$ as the following direct sum of mutually orthogonal subspaces:

$$
\operatorname{Ker}_{p}=L_{1} \oplus L_{2} \oplus \cdots \oplus L_{m} \oplus V=\left\langle X_{1}, Y_{1}, \ldots, X_{m}, Y_{m}\right\rangle \oplus V
$$

where $h_{p} v=0$ for all $v \in V$. Each $L_{i}$ is of signature $(1,1)$ because $\left\{\tilde{X}_{i}=\frac{1}{\sqrt{2}}\left(X_{i}+Y_{i}\right), \tilde{Y}_{i}=\right.$ $\left.\frac{1}{\sqrt{2}}\left(X_{i}-Y_{i}\right)\right\}$ is a pseudo-orthonormal basis such that $g_{p}\left(\tilde{X}_{i}, \tilde{X}_{i}\right)=-g_{p}\left(\tilde{Y}_{i}, \tilde{Y}_{i}\right)=g_{p}\left(X_{i}, Y_{i}\right)=$ $\pm 1, g_{p}\left(\tilde{X}_{i}, \tilde{Y}_{i}\right)=0$. Therefore, $\left\langle X_{1}, Y_{1}, \ldots, X_{m}, Y_{m}\right\rangle$ is of signature $(m, m)$ and, since $K e r \eta_{p}$ is of signature $(n, n)$, we can take a pseudo-orthonormal basis $\left\{v_{1}, \ldots, v_{n-m}, w_{1}, \ldots, w_{n-m}\right\}$ of $V$ such that $g_{p}\left(v_{i}, v_{j}\right)=\delta_{i j}$ and $g_{p}\left(w_{i}, w_{j}\right)=-\delta_{i j}$. Therefore, it suffices to define $X_{m+i}=\frac{1}{\sqrt{2}}\left(v_{i}+\right.$ $\left.w_{i}\right), Y_{m+i}=\frac{1}{\sqrt{2}}\left(v_{i}-w_{i}\right)$ to have $g_{p}\left(X_{i}, X_{i}\right)=0=g_{p}\left(Y_{i}, Y_{i}\right), g_{p}\left(X_{i}, Y_{i}\right)=1$ and $h_{p} X_{i}=h_{p} Y_{i}=0$, $i=m+1, \ldots, n$.

If $n=1$, then $\varphi_{p} X_{1}= \pm X_{1}$ and $\varphi_{p} Y_{1}=\mp Y_{1}$ follow directly from paracontact metric properties and the definition of the basis $\left\{X_{1}, Y_{1}, \ldots, X_{n}, Y_{n}\right\}$. 
It is worth mentioning that Theorem 2.1 is true only pointwise, i.e. $\operatorname{rank}\left(h_{p}\right)$ does not need to be the same for every $p \in M$. Indeed, we will see in Examples 4.3 and 4.5 that we can construct paracontact metric $(-1, \mu)$-spaces such that $h$ is zero in some points and non-zero in others.

\section{NEW EXAMPLES}

We will first present an example of $(2 n+1)$-dimensional paracontact metric $(-1,0)$-space with rank of $h$ greater than 1. This means that, together with Examples 2.2 and 2.3, we have examples of paracontact metric $(-1, \mu)$-spaces of every possible dimension and constant rank of $h$ when $\mu=0$ and $\mu=2$.

Example 4.1 ((2n+1)-dimensional paracontact metric $(-1,0)$-space with $\operatorname{rank}(h)=m \in\{2, \ldots, n\})$. Let $\mathfrak{g}$ be the $(2 n+1)$-dimensional Lie algebra with basis $\left\{\xi, X_{1}, Y_{1}, \ldots, X_{n}, Y_{n}\right\}$ such that the only non-zero Lie brackets are:

$$
\begin{aligned}
& {\left[\xi, X_{1}\right]=X_{1}+X_{2}+Y_{1}, \quad\left[\xi, Y_{1}\right]=-Y_{1}+Y_{2},} \\
& {\left[\xi, X_{2}\right]=X_{1}+X_{2}+Y_{2}, \quad\left[\xi, Y_{2}\right]=Y_{1}-Y_{2},} \\
& {\left[\xi, X_{i}\right]=X_{i}+Y_{i}, \quad i=3, \ldots, m, \quad\left[\xi, Y_{i}\right]=-Y_{i}, \quad i=3, \ldots, m,} \\
& {\left[X_{i}, X_{j}\right]= \begin{cases}\sqrt{2} X_{1}, & \text { if } i=1, j=2, \\
-\sqrt{2} X_{j} & \text { if } i=2, j=3 \ldots, m, \\
\sqrt{2}\left[\xi, X_{i}\right], & \text { if } i=1, \ldots, m, j=m+1, \ldots, n,\end{cases} } \\
& {\left[Y_{i}, Y_{j}\right]= \begin{cases}\sqrt{2}\left(-Y_{1}+Y_{2}\right), & \text { if } i=1, j=2, \\
\sqrt{2} Y_{j}, & \text { if } i=1,2, j=3, \ldots, m,\end{cases} } \\
& {\left[X_{i}, Y_{i}\right]= \begin{cases}2 \xi+\sqrt{2}\left(X_{2}+Y_{2}\right) & \text { if } i=1, \\
-2 \xi+\sqrt{2} X_{1}, & \text { if } i=2, \\
-2 \xi+\sqrt{2}\left(X_{1}-X_{2}-Y_{2}\right), & \text { if } i=3, \ldots, m, \\
-2 \xi-\sqrt{2} X_{i}, & \text { if } i=m+1, \ldots, n,\end{cases} }
\end{aligned}
$$

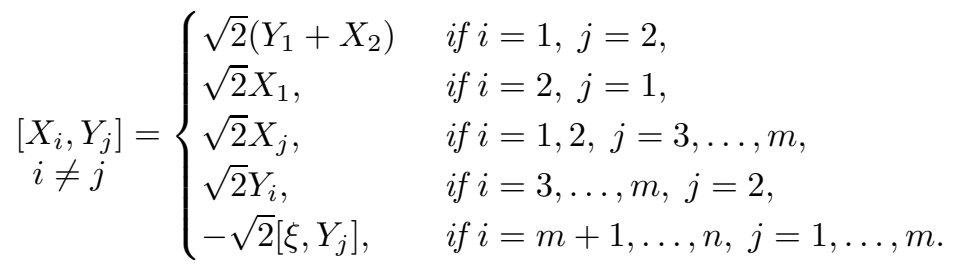

If we denote by $G$ the Lie group whose Lie algebra is $\mathfrak{g}$, we can define a left-invariant paracontact metric structure on $G$ the following way:

$$
\begin{gathered}
\varphi \xi=0, \quad \varphi X_{i}=X_{i}, \quad \varphi Y_{i}=-Y_{i}, \quad i=1, \ldots, n, \\
\eta(\xi)=1, \quad \eta\left(X_{i}\right)=\eta\left(Y_{i}\right)=0, \quad i=1, \ldots, n .
\end{gathered}
$$

The only non-vanishing components of the metric are

$$
g(\xi, \xi)=g\left(X_{1}, Y_{1}\right)=1, \quad g\left(X_{i}, Y_{i}\right)=-1, \quad i=2, \ldots, n .
$$

A straightforward computation gives that $h X_{i}=Y_{i}, i=1, \ldots, m, h X_{i}=0, i=m+1, \ldots, n$ and $h Y_{i}=0, i=1, \ldots, n$, so $h^{2}=0$ and $\operatorname{rank}(h)=m$.

Moreover, very long but direct computations give that

$$
\begin{aligned}
R\left(X_{i}, \xi\right) \xi & =-X_{i}, \quad i=1, \ldots, n, \\
R\left(Y_{i}, \xi\right) \xi & =-Y_{i}, \quad i=1, \ldots, n, \\
R\left(X_{i}, X_{j}\right) \xi & =R\left(X_{i}, Y_{j}\right) \xi=R\left(Y_{i}, Y_{j}\right) \xi=0, \quad i, j=1, \ldots, n .
\end{aligned}
$$


Therefore, the manifold is also a (-1,0)-space.

Remark 4.2. Note that the previous example is only possible when $n \geq 2$. If $n=1$, then we can only construct examples of rank $(h)=1$, as in Example 2.3.

In the definition of the Lie algebra of the previous example, some values of $i$ and $j$ are not possible for $m=2$ or $m=n$. In that case, removing the affected Lie brackets from the definition will give us valid examples nonetheless.

We will present now an example of 3-dimensional paracontact metric $(-1,2)$-space and one of 3 -dimensional paracontact metric $(-1,0)$-space, such that $\operatorname{rank}\left(h_{p}\right)=0$ or 1 depending on the point $p$ of the manifold. These are the first examples of paracontact metric $(\kappa, \mu)$-spaces with $h$ of non-constant rank that are known.

Example 4.3 (3-dimensional paracontact metric $(-1,2)$-space with $\operatorname{rank}\left(h_{p}\right)$ not constant). We consider the manifold $M=\mathbb{R}^{3}$ with the usual cartesian coordinates $(x, y, z)$. The vector fields

$$
e_{1}=\frac{\partial}{\partial x}+x z \frac{\partial}{\partial y}-2 y \frac{\partial}{\partial z}, \quad e_{2}=\frac{\partial}{\partial y}, \quad \xi=\frac{\partial}{\partial z}
$$

are linearly independent at each point of $M$. We can compute

$$
\left[e_{1}, e_{2}\right]=2 \xi, \quad\left[e_{1}, \xi\right]=-x e_{2}, \quad\left[e_{2}, \xi\right]=0 .
$$

We define the semi-Riemannian metric $g$ as the non-degenerate one whose only non-vanishing components are $g\left(e_{1}, e_{2}\right)=g(\xi, \xi)=1$, and the 1-form $\eta$ as $\eta=2 y d x+d z$, which satisfies $\eta\left(e_{1}\right)=$ $\eta\left(e_{2}\right)=0, \eta(\xi)=1$. Let $\varphi$ be the $(1,1)$-tensor field defined by $\varphi e_{1}=e_{1}, \varphi e_{2}=-e_{2}, \varphi \xi=0$. Then

$$
\begin{aligned}
d \eta\left(e_{1}, e_{2}\right) & =\frac{1}{2}\left(e_{1}\left(\eta\left(e_{2}\right)\right)-e_{2}\left(\eta\left(e_{1}\right)\right)-\eta\left(\left[e_{1}, e_{2}\right]\right)\right)=-1=-g\left(e_{1}, e_{2}\right)=g\left(e_{1}, \varphi e_{2}\right), \\
d \eta\left(e_{1}, \xi\right) & =\frac{1}{2}\left(e_{1}(\eta(\xi))-\xi\left(\eta\left(e_{1}\right)\right)-\eta\left(\left[e_{1}, \xi\right]\right)=0=g\left(e_{1}, \varphi \xi\right),\right. \\
d \eta\left(e_{2}, \xi\right) & =\frac{1}{2}\left(e_{2}(\eta(\xi))-\xi\left(\eta\left(e_{2}\right)\right)-\eta\left(\left[e_{2}, \xi\right]\right)=0=g\left(e_{2}, \varphi \xi\right) .\right.
\end{aligned}
$$

Therefore, $(\varphi, \xi, \eta, g)$ is a paracontact metric structure on $M$.

Moreover, $h \xi=0, h e_{1}=x e_{2}, h e_{2}=0$. Hence, $h^{2}=0$ and, given $p=(x, y, z) \in \mathbb{R}^{3}, \operatorname{rank}\left(h_{p}\right)=0$ if $x=0$ and $\operatorname{rank}\left(h_{p}\right)=1$ if $x \neq 0$.

Let $\nabla$ be the Levi-Civita connection. Using the properties of a paracontact metric structure and Koszul's formula

(3) $2 g\left(\nabla_{X} Y, Z\right)=X(g(Y, Z))+Y(g(Z, X))-Z(g(X, Y))-g(X,[Y, Z])-g(Y,[X, Z])+g(Z,[X, Y])$, we can compute

$$
\begin{gathered}
\nabla_{\xi} \xi=0, \quad \nabla_{e_{1}} \xi=-e_{1}-x e_{2}, \quad \nabla_{e_{2}} \xi=e_{2}, \quad \nabla_{\xi} e_{1}=-e_{1}, \quad \nabla_{\xi} e_{2}=e_{2}, \\
\nabla_{e_{1}} e_{1}=x \xi, \quad \nabla_{e_{2}} e_{2}=0, \quad \nabla_{e_{1}} e_{2}=\xi, \quad \nabla_{e_{2}} e_{1}=-\xi .
\end{gathered}
$$

Using the following definition of the Riemannian curvature

$$
R(X, Y) Z=\nabla_{X} \nabla_{Y} Z-\nabla_{Y} \nabla_{X} Z-\nabla_{[X, Y]} Z,
$$

we obtain

$$
R\left(e_{1}, \xi\right) \xi=-e_{1}+2 h e_{1}, \quad R\left(e_{2}, \xi\right) \xi=-e_{2}+2 h e_{2}, \quad R\left(e_{1}, e_{2}\right) \xi=0,
$$

so the paracontact metric manifold $M$ is also a $(-1,2)$-space.

Remark 4.4. The previous example does not contradict Theorem[2.1, as we will see by constructing explicitly the basis of the theorem on each point $p$ where $h_{p} \neq 0$, i.e., on every point $p=(x, y, z)$ such that $x \neq 0$.

Indeed, let us take a point $p=(x, y, z) \in \mathbb{R}^{3}$. If $x \neq 0$, then we define $X_{1}=\frac{e_{1 p}}{\sqrt{|x|}}, Y_{1}=\frac{h_{p} e_{1 p}}{\sqrt{|x|}}$. We obtain that $\left\{\xi_{p}, X_{1}, Y_{1}\right\}$ is a basis of $T_{p}\left(\mathbb{R}^{3}\right)$ that satisfies that: 
- the only non-vanishing components of $g$ are $g_{p}\left(\xi_{p}, \xi_{p}\right)=1, \quad g_{p}\left(X_{1}, Y_{1}\right)=\operatorname{sign}(x)$,

- the tensor $h$ can be written as $h_{p_{\mid\left\langle\xi_{p}, X_{1}, Y_{1}\right\rangle}}=\left(\begin{array}{lll}0 & 0 & 0 \\ 0 & 0 & 0 \\ 0 & 1 & 0\end{array}\right)$,

- $\varphi_{p} \xi=0, \quad \varphi_{p} X_{1}=X_{1}, \quad \varphi_{p} Y_{1}=-Y_{1}$.

Example 4.5 (3-dimensional paracontact metric $(-1,0)$-space with $\operatorname{rank}\left(h_{p}\right)$ not constant). We consider the manifold $M=\mathbb{R}^{3}$ with the usual cartesian coordinates $(x, y, z)$. The vector fields

$$
e_{1}=\frac{\partial}{\partial x}+x e^{-2 z} \frac{\partial}{\partial y}-2 y \frac{\partial}{\partial z}, \quad e_{2}=\frac{\partial}{\partial y}, \quad \xi=\frac{\partial}{\partial z}
$$

are linearly independent at each point of $M$. We can compute

$$
\left[e_{1}, e_{2}\right]=2 \xi, \quad\left[e_{1}, \xi\right]=2 x e^{-2 z} e_{2}, \quad\left[e_{2}, \xi\right]=0 .
$$

We define the semi-Riemannian metric $g$ as the non-degenerate one whose only non-vanishing components are $g\left(e_{1}, e_{2}\right)=g(\xi, \xi)=1$, and the 1 -form $\eta$ as $\eta=2 y d x+d z$, which satisfies $\eta\left(e_{1}\right)=$ $\eta\left(e_{2}\right)=0, \eta(\xi)=1$. Let $\varphi$ be the $(1,1)$-tensor field defined by $\varphi e_{1}=e_{1}, \varphi e_{2}=-e_{2}, \varphi \xi=0$. Then

$$
\begin{aligned}
d \eta\left(e_{1}, e_{2}\right) & =\frac{1}{2}\left(e_{1}\left(\eta\left(e_{2}\right)\right)-e_{2}\left(\eta\left(e_{1}\right)\right)-\eta\left(\left[e_{1}, e_{2}\right]\right)\right)=-1=-g\left(e_{1}, e_{2}\right)=g\left(e_{1}, \varphi e_{2}\right), \\
d \eta\left(e_{1}, \xi\right) & =\frac{1}{2}\left(e_{1}(\eta(\xi))-\xi\left(\eta\left(e_{1}\right)\right)-\eta\left(\left[e_{1}, \xi\right]\right)=0=g\left(e_{1}, \varphi \xi\right),\right. \\
d \eta\left(e_{2}, \xi\right) & =\frac{1}{2}\left(e_{2}(\eta(\xi))-\xi\left(\eta\left(e_{2}\right)\right)-\eta\left(\left[e_{2}, \xi\right]\right)=0=g\left(e_{2}, \varphi \xi\right) .\right.
\end{aligned}
$$

Therefore, $(\varphi, \xi, \eta, g)$ is a paracontact metric structure on $M$.

Moreover, $h \xi=0, h e_{1}=-2 x e^{-2 z} e_{2}$, he $e_{2}=0$. Hence, $h^{2}=0$ and, given $p=(x, y, z) \in \mathbb{R}^{3}$, $\operatorname{rank}\left(h_{p}\right)=0$ if $x=0$ and $\operatorname{rank}\left(h_{p}\right)=1$ if $x \neq 0$.

Let $\nabla$ be the Levi-Civita connection. Using the properties of a paracontact metric structure and Koszul's formula (3), we can compute

$$
\begin{gathered}
\nabla_{\xi} \xi=0, \quad \nabla_{e_{1}} \xi=-e_{1}+2 x e^{-2 z} e_{2}, \quad \nabla_{e_{2}} \xi=e_{2}, \quad \nabla_{\xi} e_{1}=-e_{1}, \quad \nabla_{\xi} e_{2}=e_{2}, \\
\nabla_{e_{1}} e_{1}=-2 x e^{-2 z} \xi, \quad \nabla_{e_{2}} e_{2}=0, \quad \nabla_{e_{1}} e_{2}=\xi, \quad \nabla_{e_{2}} e_{1}=-\xi .
\end{gathered}
$$

Using now (4), we obtain

$$
R\left(e_{1}, \xi\right) \xi=-e_{1}, \quad R\left(e_{2}, \xi\right) \xi=-e_{2}, \quad R\left(e_{1}, e_{2}\right) \xi=0
$$

so the paracontact metric manifold $M$ is also a $(-1,0)$-space.

Acknowledgements. The author would like to thank Prof. Martín Avendaño for his invaluable help.

\section{REFERENCES}

[1] B. Cappelletti Montano, Bi-Legendrian structures and paracontact geometry, Int. J. Geom. Met. Mod. Phys. 6 (2009) 487-504.

[2] B. Cappelletti Montano, Bi-paracontact structures and Legendre foliations, Kodai Math. J. 33 (2010) $473-512$.

[3] B. Cappelletti Montano, A. Carriazo, V. Martín-Molina, Sasaki-Einstein and paraSasaki-Einstein metrics from $(\kappa, \mu)$-structures, J. Geom. Physics. 73 (2013) 20-36.

[4] B. Cappelletti Montano, L. Di Terlizzi, Geometric structures associated with a contact metric $(\kappa, \mu)$-space, Pacific J. Math. 246 (2010) 257-292.

[5] B. Cappelletti Montano, I. Küpeli Erken, C. Murathan, Nullity conditions in paracontact geometry, Differential Geom. Appl. 30 (2012) 665-693.

[6] G. Calvaruso, Homogeneous paracontact metric three-manifolds, Illinois J. Math. 55 (2011) 697-718.

[7] G. Calvaruso, V. Martín-Molina, Paracontact metric structures on the unit tangent sphere bundle, to appear in Ann. Mat. Pura Appl. (4), DOI: 10.1007/s10231-014-0424-4.

[8] G. Calvaruso, D. Perrone, Geometry of $H$-paracontact metric manifolds, arXiv:1307.7662

[9] S. Ivanov, D. Vassilev, S. Zamkovoy, Conformal paracontact curvature and the local flatness theorem, Geom. Dedicata 144 (2010) 79-100. 
[10] S. Kaneyuki, F. L. Williams, Almost paracontact and parahodge structures on manifolds, Nagoya Math. J. 99 (1985) 173-187.

[11] V. Martín-Molina, Paracontact metric manifolds without a contact metric counterpart, to appear in Taiwanese J. Math., DOI: 10.11650/tjm.18.2014.4447.

[12] C. Murathan, I. Küpeli Erken, A Complete Study of Three-Dimensional Paracontact $(\kappa, \mu, \nu)$-spaces, arXiv:1305.1511 3.

[13] B. O'Neill. Semi-Riemannian Geometry with applications to relativity, Academic Press, New York, 1983.

[14] S. Zamkovoy, Canonical connections on paracontact manifolds, Ann. Glob. Anal. Geom. 36 (2009) 37-60.

[15] S. Zamkovoy, V. Tzanov, Non-existence of flat paracontact metric structures in dimension greater than or equal to five, Annuaire Univ. Sofia Fac. Math. Inform. 100 (2011) 27-34.

E-mail address: vmartin@unizar.es

Centro Universitario de la Defensa de Zaragoza, Academia General Militar, Ctra. de Huesca s/n, 50090 Zaragoza, SPAIN, AND I.U.M.A, Universidad de Zaragoza, SPAIN 